\title{
Long-term safety, efficacy and inhibition of radiographic progression with abatacept treatment in patients with rheumatoid arthritis and an inadequate response to methotrexate: 3-year results from the AIM trial
}

\author{
Joel M Kremer, ${ }^{1}$ Anthony S Russell, ${ }^{2}$ Paul Emery, ${ }^{3,4}$ Carlos Abud-Mendoza, ${ }^{5}$ \\ Jacek Szechinski, ${ }^{6}$ Rene Westhovens, ${ }^{7}$ Tracy Li, ${ }^{8}$ Xianhuang Zhou, ${ }^{8}$ \\ Jean-Claude Becker, ${ }^{8}$ Richard Aranda, ${ }^{8}$ Charles Peterfy, ${ }^{9}$ Harry K Genant ${ }^{10}$
}

\begin{abstract}
- An additional figure is published online only. To view the file please visit the journal online (http://ard.bmj.com)
\end{abstract}

${ }^{1}$ Center for Rheumatology, Albany Medical College, Albany, New York, USA

2University of Alberta Hospital, Edmonton, Alberta, Canada

${ }^{3}$ Section of Musculoskeletal Disease, Leeds Institute of Molecular Medicine, University of Leeds, Leeds, UK ${ }^{4} \mathrm{NIHR}$ Leeds Musculoskeletal Biomedical Research Unit, Leeds Teaching Hospitals Trust, Leeds, UK

${ }^{5}$ Regional Unit of Rheumatology, Faculty of Medicine and Central Hospital, University of San Luis Potosí, México

${ }^{6}$ Department of Rheumatology, Medical University of Wroclaw, Wroclaw, Poland

${ }^{7}$ Department of Rheumatology, Universitaire Ziekenhuizenn Leuven, Leuven, Belgium ${ }^{8}$ Bristol-Myers Squibb, Princeton, New Jersey, USA ${ }^{9}$ Spire Sciences LLC, San Francisco, California, USA ${ }^{10}$ UCSF/Synarc, San Francisco, California, USA

\section{Correspondence to} Joel M Kremer, Center for Rheumatology, Albany Medical College, 1367 Washington Ave, Albany, NY 12206, USA; jkremer@joint-docs.com

Accepted 11 June 2011

\section{ABSTRACT}

Objective To evaluate abatacept treatment over 3 years in patients with rheumatoid arthritis (RA) refractory to methotrexate (MTX).

Methods Patients randomised to abatacept or placebo (+MTX) during the 1-year double-blind period of the Abatacept in Inadequate responders to Methotrexate (AIM) trial received open-label abatacept (+MTX) in the long-term extension (LTE). Safety was assessed for patients who received $\geq 1$ dose of abatacept, regardless of randomisation group. Efficacy was assessed for patients randomised to abatacept who entered the LTE.

Results 433 and 219 patients were randomised and treated with abatacept or placebo, respectively; 378 and 161 entered the LTE. At year 3, 440/539 patients were ongoing. No unexpected safety events were observed in the LTE. By year 3, incidence rates of adverse event and serious adverse events were 249.8/100 and 15.1/100 patient-years, respectively. Incidence rates were generally stable over time. At year 3, 84.8\%, $63.4 \%$ and $37.5 \%$ of patients achieved American College of Rheumatology (ACR) criteria of 20, 50 and 70 , respectively, compared with $82.3 \%, 54.3 \%$ and $32.4 \%$ of patients at year 1 . Mean changes in Genant-modified Sharp scores were reduced progressively over 3 years, with significantly greater inhibition during year 3 compared with year 2 $(p=0.022$ for total score).

Conclusion In MTX-inadequate responders with RA, abatacept provided consistent safety and sustained efficacy over 3 years. The data suggest an increasing inhibitory disease-modifying effect on radiographic progression.

\section{INTRODUCTION}

Rheumatoid arthritis (RA) is a chronic disorder requiring long-term treatment. As joint damage in patients with RA is often progressive and patients remain on treatment for extensive periods, long-term follow-up is required to assess efficacy and safety of interventions. In the phase III AIM (Abatacept in Inadequate responders to Methotrexate (MTX)) trial, ${ }^{1}$ abatacept demonstrated sustained clinical efficacy and consistent safety over 2 years of treatment. ${ }^{2}$ An increasing inhibition of radiographic progression was also reported over each year. ${ }^{3}$ In this paper we report the safety, efficacy, inhibition of radiographic progression and impact on health-related quality of life (HRQoL) of abatacept over 3 years.

\section{METHODS}

\section{Study design}

Patients who completed the double-blind period of the AIM trial ${ }^{1}$ were eligible to enter the openlabel long-term extension (LTE), where patients originally randomised $2: 1$ to either abatacept or placebo (+MTX) received open-label abatacept (approximately $10 \mathrm{mg} / \mathrm{kg}$ according to weight range) every 28 days. $^{2}$ During the LTE, patients continued taking MTX; adjustments to MTX dose were permitted in addition to other concomitant medications, including background diseasemodifying antirheumatic drugs (DMARDs). The cumulative period included the 1-year doubleblind period plus 2 years of the LTE ( 3 years in total). During the first 12 months both patients and physicians were blinded to treatment assignment; radiologists were blinded to the treatment and order of time points throughout.

\section{Safety assessments}

Safety was reported according to the Medical Dictionary for Regulatory Activities. For patients who prematurely discontinued, adverse events (AEs) were recorded at days 28,56 and 85 after the last abatacept dose.

\section{Clinical efficacy, physical function and HROOL assessments}

Clinical efficacy was assessed using the American College of Rheumatology (ACR) criteria and Disease Activity Score in 28 joints (DAS28, based on $\mathrm{C}$ reactive protein (CRP) levels; scale of 0-9.3). Physical function and HRQoL were evaluated using the Health Assessment Questionnaire-Disability Index (HAQ-DI; scale of $0-3$, response defined as a decrease of $\geq 0.3$ from baseline) and Short Form (SF)-36 (scale of 0-100, clinically meaningful improvement defined as change of $\geq 3$ units from baseline), respectively, as previously described. ${ }^{1}$ 


\section{Radiographic assessments}

Radiographic assessments were performed at baseline and years 1,2 and 3 (or within $\leq 28$ days of discontinuation). ${ }^{3}$ At year 3 , radiographs from baseline and years 1 and 2 were re-read in random order by two independent readers, blinded to treatment and order of time points. The Genant-modified Sharp scoring system assessed radiographs of joints in the hands, wrists and feet for changes in total score (TS; non-progression defined as mean change from baseline of $\leq 0)$, erosion score (ES) and joint space narrowing (JSN) score (maximum scores 290, 145 and 145, respectively). ${ }^{2}$

\section{Statistical analysis}

Safety analyses included all patients who received $\geq 1$ dose of abatacept in the cumulative period, regardless of randomisation group (abatacept or placebo). Incidence rates (IRs)/100 patientyears of exposure were calculated as previously described. ${ }^{2}$

Clinical efficacy and radiographic progression are presented for patients originally randomised to abatacept who entered the LTE and received $\geq 1$ dose of abatacept, with data available at the visit of interest (as-observed). For assessment of the ACR data set, the tender and swollen joint counts and five components were scored as ' 0 ' when the patient did not satisfy a component criterion, ' 1 ' when the patient did satisfy a component criterion or missing ('-') when no data were available. Patients were only included in the as-observed analysis for each ACR response (ACR 20, 50 or 70) when they had either joints of ' 1 ' and at least three (of the five) components of ' 1 ' or joints of ' 0 ' and at least three (of the five) components of ' 0 '. Thus, for every patient on every visit day, ACR 20, 50 and 70 responses were assessed independently, resulting in different numbers for ACR responses at each given time point.

For mean changes in radiographic scores, all patients with radiographs at baseline who received $\geq 1$ dose of abatacept in year 2 were included in the year 3 analysis. Patients who discontinued during the LTE were requested to return for follow-up radiography at their next annual visit, regardless of subsequent prescribed treatment. Radiographs were taken at the time of discontinuation in those patients who declined to return for an annual visit and their next annual assessment imputed with linear extrapolation based on the last available annual visit and assessment at discontinuation. A non-parametric analysis was performed to compare the distribution of mean change scores (TS, ES and JSN score). ${ }^{2} 3$

\section{RESULTS}

\section{Patient disposition, baseline characteristics and demographics}

Of the 433 and 219 patients randomised to abatacept and placebo, respectively, $82.3 \%$ and $80.1 \%$ of these were ongoing at year 3 . For the 433 randomised abatacept-treated patients, the retention rate at year 3 was $72 \%$ with $11.1 \%$ (48/433), $12.2 \%(46 / 378)$ and $6.3 \%(21 / 332)$ of patients discontinuing, respectively, during years 1, 2 and 3; 5.8\% (25/433) and $8.8 \%$ (38/433) of these patients discontinued due to lack of efficacy and AEs, respectively, over the 3 years (see figure 1 in online supplement).

Baseline demographics and clinical characteristics of patients entering the LTE were comparable between treatment groups, as previously reported. ${ }^{12}$ Overall, mean age and disease duration were 50.8 and 8.5 years, respectively. The mean (SD) Genantmodified Sharp TS was 45.2 (35.1) and DAS28 (CRP) was 6.4 (0.8). All patients received concomitant MTX during the study. For patients randomised to abatacept who entered the LTE, mean MTX doses decreased from $15.1 \mathrm{mg}$ at baseline to 14.4 , 14.3 and $14.2 \mathrm{mg}$ at years 1,2 and 3, respectively.

\section{Safety}

Overall safety

During the 3-year cumulative period mean abatacept exposure was 34.3 months. Of patients originally randomised to abatacept, a total of 569 patients $(96 \%)$ experienced an AE with an IR of 249.8/100 patient-years; a similar IR was reported in the double-blind period (table 1). The type and incidence of the most commonly reported AEs during the cumulative period were similar to those reported in the double-blind period, ${ }^{1}{ }^{2}$ as were the IRs of serious AEs (SAEs). In the cumulative period, 55 and 36 patients discontinued due to AEs and SAEs, respectively. Cardiac disorders were reported in 68 patients $(11.4 \%)$ during the cumulative period at an IR of $4.37 / 100$ patient-years.

Ten deaths were reported in the cumulative period in patients who had received $\geq 1$ dose of abatacept; bronchopulmonary aspergillosis in year $1(n=1)$, myocardial ischaemia with postprocedural complications, lobar pneumonia and lung cancer in year $2(n=1$ for all) and, in year 3, pneumonia/sepsis, malignant melanoma and aortic aneurysm ( $\mathrm{n}=1$ each) and cardiac arrest $(\mathrm{n}=3)$.

\section{Infection}

IRs of infections and serious infections were lower in the cumulative versus the double-blind period (table 1). Two cases of tuberculosis were documented in the cumulative period, both in the abatacept group; both patients discontinued. One case was suspected in a 55-year-old woman in the double-blind period. The patient did not experience any symptoms and no bacterial evidence of tuberculosis was found. During the LTE, tuberculosis was diagnosed presumptively in a 39-year-old woman. The patient exhibited a response to tuberculosis therapy, although bronchial lavage and biopsy did not identify tuberculosis.

\section{Malignancy}

During the cumulative period, malignant neoplasms were reported in 24 patients (1.5 events/100 patient-years; table 1). The three cases of lung cancer were reported during the LTE in patients initially randomised to abatacept; all had a history of

Table 1 Summary of adverse events and malignancy type for all patients who received one dose of abatacept during 3 years of the study

\begin{tabular}{lll}
\hline Event, IR/100 patient-years & $\begin{array}{l}\text { Double-blind period } \\
\text { abatacept+ MTX } \\
\text { (n=433) }\end{array}$ & $\begin{array}{l}\text { Cumulative period* } \\
\text { abatacept+ MTX } \\
\text { (n=594) }\end{array}$ \\
\hline Total AEs & 303.4 & 249.8 \\
Total SAEs & 17.7 & 15.1 \\
Infections & 90.5 & 70.8 \\
Serious infections & 4.2 & 3.2 \\
Malignancies & 1.7 & 1.5 \\
Autoimmune events & 2.5 & 2.2 \\
Malignancy type, $\mathrm{n}$ & & \\
Total patients with malignancies & 7 & 24 \\
Non-melanoma skin cancer & 5 & 15 \\
Basal cell carcinoma & 4 & 12 \\
Squamous cell carcinoma & 1 & 3 \\
Solid organ & 0 & 6 \\
Lung & 0 & 3 \\
Prostate & 0 & 1 \\
Endometrial & 0 & 1 \\
Melanoma & 0 & 1 \\
Haematological & 2 & 3 \\
Lymphoma & 1 & 2 \\
Myelodysplastic syndrome & 1 & 1 \\
\hline Data & 5 & 1 \\
\hline
\end{tabular}

* Data are presented for all patients who participated in the long-term extension regardless of randomised double-blind treatment group.

$A E$, adverse event; IR, incidence rate; MTX, methotrexate; SAE, serious adverse event. 

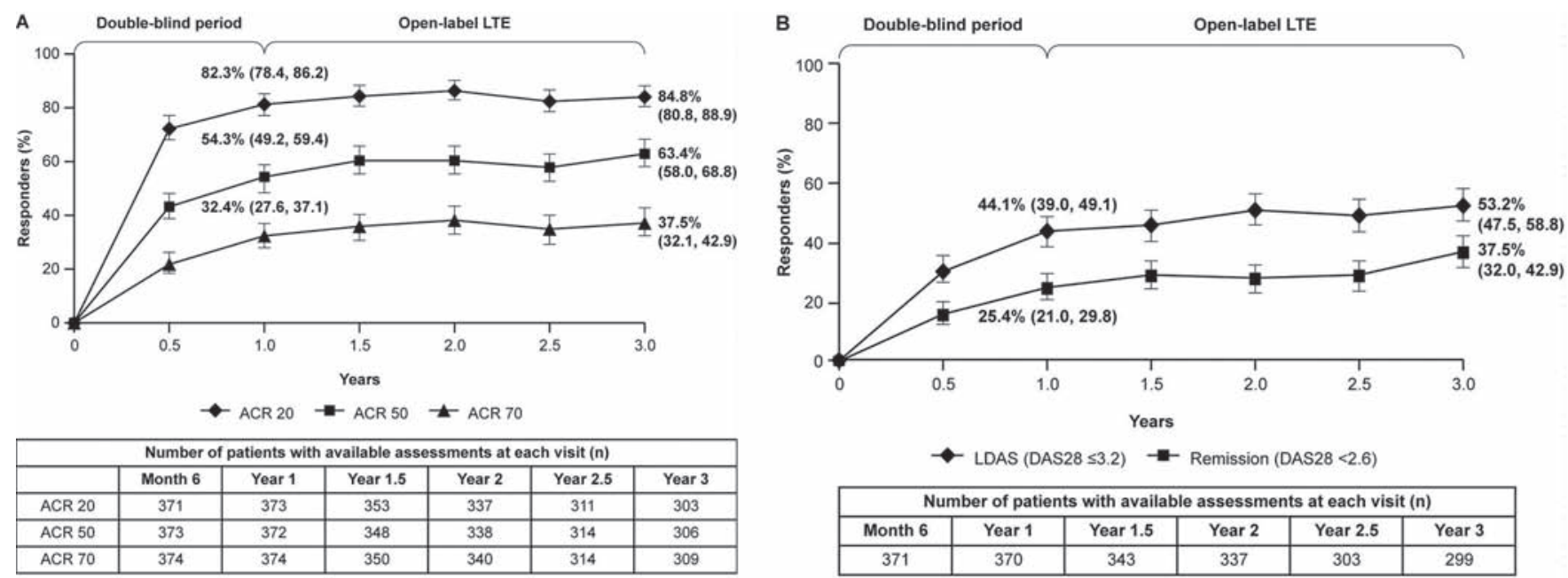

\begin{tabular}{|l|c|c|c|c|c|c|}
\hline \multicolumn{7}{|c|}{ Number of patients with available assessments at each visit (n) } \\
\hline & Month 6 & Year 1 & Year 1.5 & Year 2 & Year 2.5 & Year 3 \\
\hline ACR 20 & 371 & 373 & 353 & 337 & 311 & 303 \\
\hline ACR 50 & 373 & 372 & 348 & 338 & 314 & 306 \\
\hline ACR 70 & 374 & 374 & 350 & 340 & 314 & 309 \\
\hline
\end{tabular}
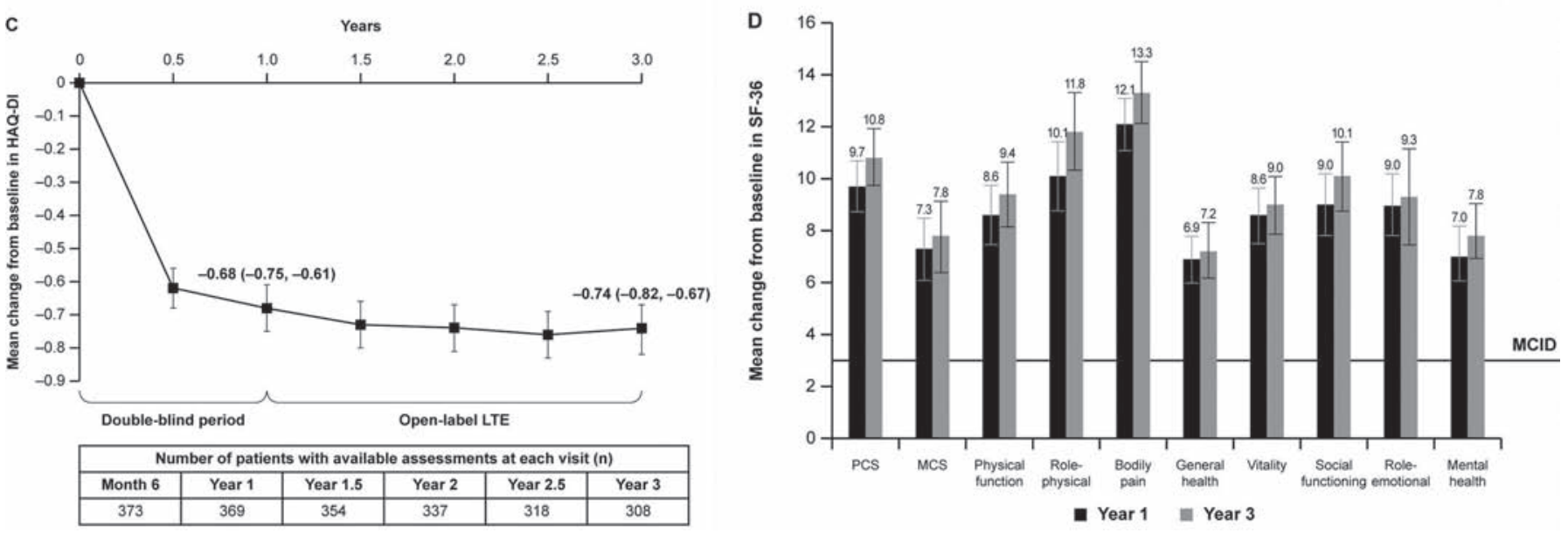

Figure 1 Clinical efficacy and patient-reported outcomes in abatacept-treated patients. (A) Proportion of patients achieving American College of Rheumatology (ACR) 20, 50 and 70 responses over 3 years. (B) Proportion of patients achieving low disease activity state (LDAS) and Disease Activity Score 28 (DAS28) (C reactive protein (CRP))-defined remission over 3 years. (C) Mean change from baseline in Health Assessment Questionnaire-Disability Index (HAQ-DI) over 3 years. (D) Mean change from baseline in the physical component summary (PCS), mental component summary (MCS) and Short Form (SF)-36 individual subscales at years 1 and 3. Data are presented with $95 \%$ Cls for patients randomised to abatacept who entered the long-term extension (LTE) to receive $\geq 1$ dose of abatacept, with available assessments at the visit of interest (as-observed population). LDAS defined as DAS28 (CRP) $\leq 3.2$. DAS28defined remission defined as DAS28 (CRP) <2.6. At year 1, 370, 370, 373, 372, 373, 373, 373, 373, 370 and 373 patients were included in the PCS, MCS, physical function, role-physical, bodily pain, general health, vitality, social functioning, role-emotional and mental health assessments, respectively. At year 3, 308, 308, 310, 309, 309, 312, 312, 312, 309 and 312 patients were included in the aforementioned assessments, respectively. A change of 3.0 units in MCS and PCS was considered the minimum clinically important difference (MCID).

smoking. Of the two cases of lymphoma, one was reported during the double-blind period (malignant thyroid tumour of moderate intensity). The second case (non-Hodgkin's lymphoma) was reported during the first year of the LTE in a patient randomised to placebo.

\section{Autoimmune events}

During the cumulative period, 35 autoimmune AEs were reported. The most common were psoriasis, Sjögren's syndrome and vasculitis (12, 4 and 4 patients, respectively).

\section{Efficacy}

ACR responses

For patients originally randomised to abatacept, the proportions of ACR 20, 50 and 70 responders were maintained from year 1 to year 3 (figure $1 \mathrm{~A}$ ).

\section{Disease activity}

The proportion of patients achieving low disease activity state or DAS28-defined remission in the original abatacept-treated group increased over 3 years (figure 1B). For DAS28-defined remission, 95\% CI did not overlap for the year 1 and 3 assessments (figure 1B). Mean (SD) DAS28 (CRP) at year 3 was 3.16 (1.18), with a mean (SE) change from baseline of -3.21 (0.08).

Physical function and $\mathrm{HROOL}$

At baseline, patients randomised to abatacept had a mean (SD) HAO-DI score of 1.7 (0.6). A clinically meaningful improvement in physical function (HAQ-DI response) was achieved in $71.8 \%$ (265/369; 95\% CI 67.2 to 76.4 ) of these patients at year 1; a comparable proportion achieved a HAO-DI response at year 3 (73.1\% (255/308); $95 \%$ CI 68.1 to 78.0$)$. Mean changes from baseline are shown in figure $1 \mathrm{C}$. 

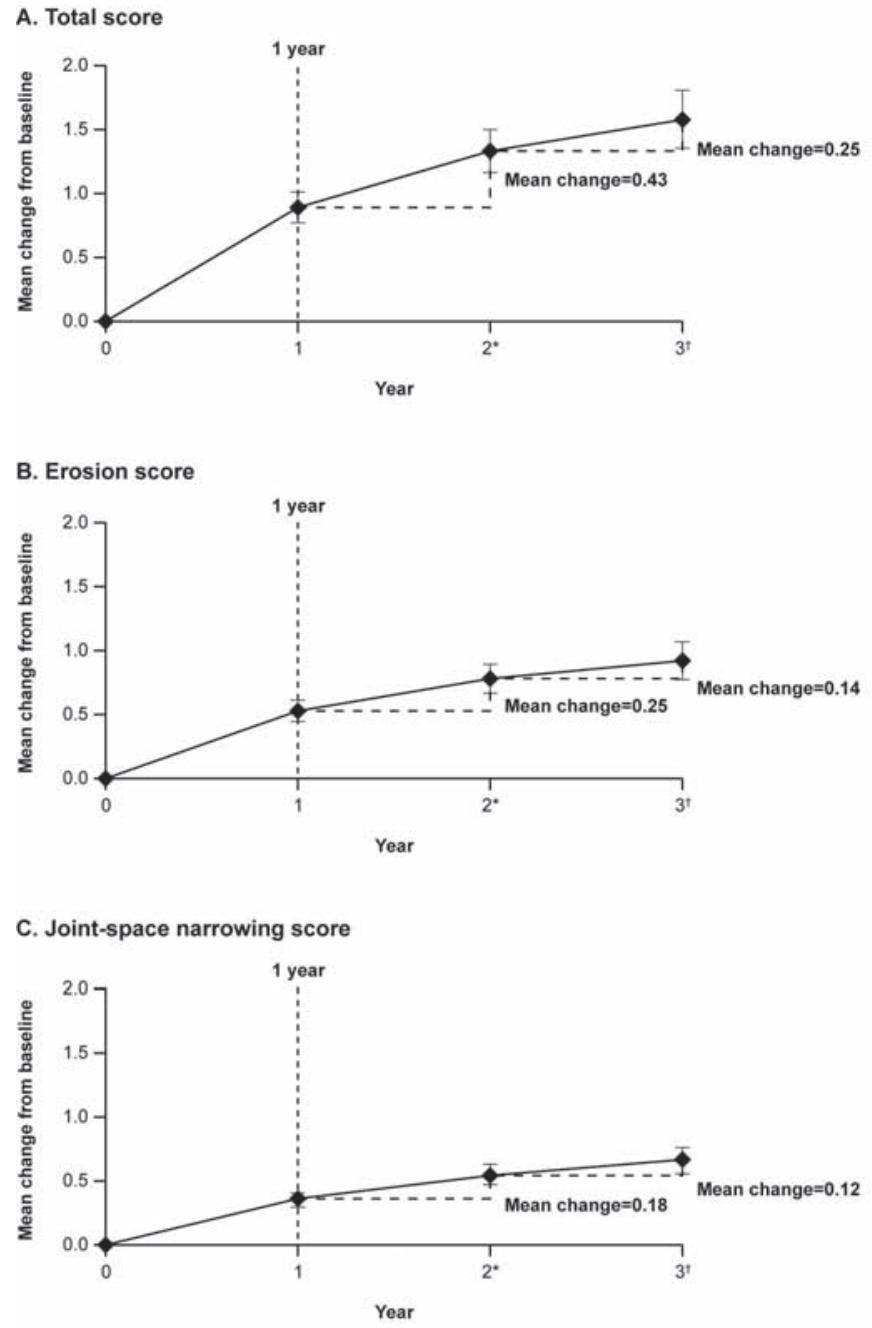

D.

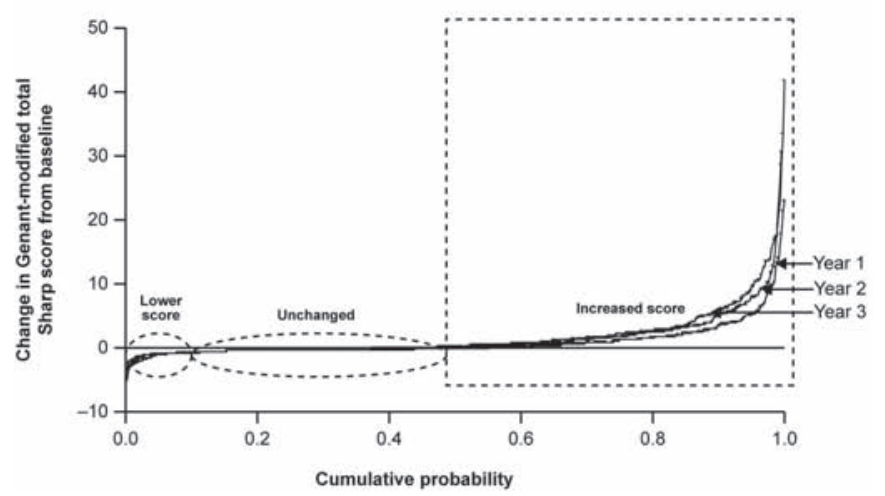

Figure 2 Impact on radiographic disease progression over 3 years of abatacept treatment. (A) Mean change from baseline in Genantmodified Sharp total score. (B) Mean change in erosion score. (C) Mean change in joint space narrowing score. (D) Cumulative probability plot showing change from baseline in total score. Data based on all patients randomised to abatacept who entered the long-term extension to receive $\geq 1$ dose of abatacept, from year 1-2 $(n=297)^{*}$ and year $2-3(n=295) \dagger$. Error bars represent the SEM.

At year 3 of abatacept treatment, a clinically meaningful improvement of $>3$ units was observed in the summary scores and individual subscales of the SF-36. Mean improvements from baseline in the physical component summary (PCS) and mental component summary (MCS) at year 1 were maintained over 3 years (figure 1D). Of the SF-36 subscales, the greatest improvement was observed in bodily pain and role-physical from baseline to years 1 and 3 (figure 1D).

\section{Radiographic progression}

A total of $304 / 378$ (81\%), 293/378 (78\%) and 302/378 (80\%) abatacept-treated patients had radiographs available at baseline and at years 1,2 and 3, respectively, and were assessed. Mean changes from baseline in Genant-modified Sharp scores were progressively reduced from baseline to years 1, 2 and 3 (figure 2A-C). Overall, scores were reduced by approximately $50 \%$ in year 2 relative to year 1 . Progression slowed even further between years 2 and 3 , with a mean change in TS of 0.25 $(p=0.022$ for rate of progression in year 1 to year 2 vs year 2 to year 3).

At year 1, 46\% (139/304) of patients with evaluable radiographs were considered non-progressors (change from baseline in $\mathrm{TS} \leq 0)$. Of these 139 patients, 135 had evaluable radiographs at years 1 and 2; 68\% (92/135) remained non-progressors at year 2. Of the 92 patients with no progression at year 2, 91 patients had evaluable radiographs at years 2 and 3 , of whom $76 \%$ (69/91) remained non-progressors at year 3. Overall, at year 3, $40 \%$ of patients had no radiographic progression from baseline. Cumulative probability plots of the distribution of change in TS over 3 years, at yearly intervals, show an improvement in radiographic progression with abatacept over time (figure $2 \mathrm{D}$ ). The proportion of patients with no radiographic progression increased during each year of abatacept treatment.

\section{DISCUSSION}

Retention rates in long-term clinical trials provide an indication of the likelihood of patients continuing treatment over the long term in clinical practice. In the AIM trial in patients with RA with an inadequate response to MTX, high retention rates were observed over 3 years of abatacept treatment (approximately $70 \%$ ) with low discontinuation rates due to loss of efficacy and safety. The yearly discontinuation rate decreased during year 3 , indicating the likelihood of patients to remain on treatment over the long term. ${ }^{4}$ Retention rates observed in anti-tumour necrosis factor (TNF) therapy trials have been $61-75 \%$ over $2-3$ years. $^{5-7}$

IRs of SAEs, infections, serious infections, malignancies and autoimmune events reported during long-term abatacept exposure (cumulative period for all patients who received $\geq 1$ dose of abatacept regardless of original double-blind randomisation group) were comparable with, or lower than, those observed during short-term abatacept exposure (double-blind period). Additionally, no unique or unexpected safety signals were detected with long-term exposure. Although it is considered inappropriate to make cross-trial comparisons of IRs, it may be noted that IRs of AEs and SAEs in this trial were within the lower range of IRs reported for other biologic therapies (such as TNF inhibitors) in similar patient populations. ${ }^{6} 8-10$ The overall IR of cancer in the cumulative period was $1.5 / 100$ patient-years. This is consistent with previously reported IRs of malignancy in another trial of abatacept in established disease $^{11}$ and in comparator RA cohorts treated with conventional DMARDs (0.67-1.77/100 patient-years). ${ }^{12}$

Improvements in disease status, HRQoL, physical function and radiographic inhibition were sustained over 3 years in a high proportion of patients who remained in the study and were treated with abatacept throughout this time. Despite the long disease duration and high disease burden at baseline, one-third 
achieved DAS28-defined remission and a 70\% improvement in signs and symptoms, while more than half of patients had low disease activity by year 3. For DAS28-defined remission, 95\% CIs did not overlap at years 1 and 3 , suggesting a significant improvement between these time points. In addition, at year 3, nearly three-quarters of patients had achieved a clinically meaningful improvement in physical function, and improvements in the SF-36 (MCS, PCS and all eight subscales) exceeded the minimum clinically important difference of 3 units.

Increasing inhibition of radiographic progression with abatacept treatment has previously been reported for each year of treatment over 2 years in the present study. ${ }^{3}$ Mean change in TS was reduced by approximately $50 \%$ in year 2 relative to year 1 ; further significant inhibition was observed at year 3 relative to year 2. Importantly, the majority of patients with no radiographic progression at year 2 remained free from progression at year 3 . Evaluation of changes in radiographic progression by cumulative probability shows that the proportion of patients who showed either no radiographic deterioration or some improvement increased during each year of abatacept treatment.

LTE studies should be considered in the context of their limitations, as recently highlighted by Buch et al. ${ }^{13}$ In this study, baseline demographics, although typical for this type of analysis, indicate a population with more active disease than is generally observed in clinical practice. Additionally, efficacy analyses were based on as-observed analyses, which are vulnerable to the drop-out of patients who respond less well to treatment. However, discontinuations during the LTE of this trial were relatively low, and only $3.7 \%$ of patients discontinued due to lack of efficacy.

\section{CONCLUSION}

Overall, these data show that abatacept has an increasing diseasemodifying effect on structural damage over time in the majority of patients who remain on treatment. Combined with the sustained clinical benefits, high patient retention rate and consistent safety profile observed over 3 years, these data support the long-term use of abatacept in patients with an inadequate response to MTX. This population continues to be monitored in the LTE of this trial.

Acknowledgements The authors thank Medicus International for their editorial assistance.

Funding This study and the editorial support provided were funded by Bristol-Myers Squibb.

Competing interests JMK has received research grants from Abbott, Amgen, BristolMyers Squibb, Centocor, Genentech, Merck, Pfizer, Roche and Human Genome Sciences and consulting fees from Abbott, Amgen, Bristol-Myers Squibb, Centocor, Genentech, Merck, Pfizer and Roche. ASR has received corporate funding and honoraria and has served on a speaker's bureau for Bristol-Myers Squibb, Pfizer, Hoffman-La Roche, Amgen/ Wyeth and UCB Canada. PE provided expert advice and has undertaken clinical trials for Amgen, Schering-Plough, Centocor, Bristol-Myers Squibb and Roche. CA-M has nothing to disclose. JS has received consulting fees, speaking fees and/or honoraria (less than $\$ 10000$ each) from Bristol-Myers Squibb, Roche, Wyeth and Sanofi-Aventis. RW has received consulting fees from Bristol-Myers Squibb, Schering-Plough and Roche, has served on a speaker's bureau for Bristol-Myers Squibb and Schering-Plough and has received research support from UCB. TL is a full-time employee of and stockholder in Bristol-Myers Squibb. XZ is a full-time employee of Bristol-Myers Squibb. J-CB is a full-time employee of and stockholder in Bristol-Myers Squibb. RA is a full-time employee of and stockholder in Bristol-Myers Squibb. CP is the founder and CEO of Spire Sciences which provides imaging services to multiple pharmaceutical companies. HKG has received research grants from Bristol-Myers Squibb, Roche, Genentech, Amgen, Merck, Pfizer, Servier and Biogen Idec, has received consulting fees from Bristol-Myers Squibb, Roche, Genentech, Amgen, Merck and Synarc and owns stock options in Synarc.

Ethics approval This study was conducted with the approval of the Institutional Review Board/Independent Ethics Committee.

Provenance and peer review Not commissioned; externally peer reviewed.

\section{REFERENCES}

1. Kremer JM, Genant HK, Moreland LW, et al. Effects of abatacept in patients with methotrexate-resistant active rheumatoid arthritis: a randomized trial. Ann Intern Med 2006;144:865-76.

2. Kremer JM, Genant HK, Moreland LW, et al. Results of a two-year followup study of patients with rheumatoid arthritis who received a combination of abatacept and methotrexate. Arthritis Rheum 2008;58:953-63.

3. Genant HK, Peterfy CG, Westhovens R, et al. Abatacept inhibits progression of structural damage in rheumatoid arthritis: results from the long-term extension of the AIM trial. Ann Rheum Dis 2008;67:1084-9.

4. Westhovens R, Kremer JM, Moreland LW, et al. Safety and efficacy of the selective costimulation modulator abatacept in patients with rheumatoid arthritis receiving background methotrexate: a 5 -year extended phase IIB study. J Rheumatol 2009;36:736-42.

5. Breedveld FC, Weisman MH, Kavanaugh AF, et al. The PREMIER study: a multicenter, randomized, double-blind clinical trial of combination therapy with adalimumab plus methotrexate versus methotrexate alone or adalimumab alone in patients with early, aggressive rheumatoid arthritis who had not had previous methotrexate treatment. Arthritis Rheum 2006;54:26-37.

6. Klareskog L, Gaubitz M, Rodriguez-Valverde V, et al.; Etanercept Study 301 Investigators. A long-term, open-label trial of the safety and efficacy of etanercept (Enbrel) in patients with rheumatoid arthritis not treated with other diseasemodifying antirheumatic drugs. Ann Rheum Dis 2006;65:1578-84.

7. van der Heijde D, Klareskog L, Rodriguez-Valverde V, et al.; TEMPO Study Investigators. Comparison of etanercept and methotrexate, alone and combined, in the treatment of rheumatoid arthritis: two-year clinical and radiographic results from the TEMPO study, a double-blind, randomized trial. Arthritis Rheum 2006;54:1063-74.

8. Kremer JM, Weinblatt ME, Bankhurst AD, et al. Etanercept added to background methotrexate therapy in patients with rheumatoid arthritis: continued observations. Arthritis Rheum 2003;48:1493-9.

9. Schiff M, Keiserman M, Codding C, et al. Efficacy and safety of abatacept or infliximab vs placebo in ATTEST: a phase III, multi-centre, randomised, double-blind, placebo-controlled study in patients with rheumatoid arthritis and an inadequate response to methotrexate. Ann Rheum Dis 2008;67:1096-103.

10. Schiff MH, Burmester GR, Kent JD, et al. Safety analyses of adalimumab (HUMIRA) in global clinical trials and US postmarketing surveillance of patients with rheumatoid arthritis. Ann Rheum Dis 2006;65:889-94.

11. Kremer $\mathbf{K}$, Westhovens $\mathrm{R}$, Luggen $\mathbf{M}$, et al. Long-term efficacy and safety of Abatacept through 3 years of treatment in rheumatoid arthritis patients in the AIM and ATTAIN trials. Arthritis Rheum 2007;56(Suppl 9):S300, Abstract 699.

12. Simon TA, Smitten AL, Franklin J, et al. Malignancies in the rheumatoid arthritis abatacept clinical development programme: an epidemiological assessment. Ann Rheum Dis 2009;68:1819-26.

13. Buch $\mathbf{M H}$, Aletaha D, Emery $\mathrm{P}$, et al. Reporting of long-term extension studies: lack of consistency calls for consensus. Ann Rheum Dis 2011;70:886-90. 